\title{
FROM TELESCOPE TO MPC: ORGANIZING THE MINOR
}

\section{PLANETS}

\author{
B.G. MARSDEN \\ Harvard-Smithsonian Center for Astrophysics \\ Cambridge, MA 02138 \\ U.S.A.
}

\section{Introduction}

Somewhat more than a century after its introduction for the purpose of discovering minor planets, photography is now rapidly giving way to the CCD as the technology of choice for observing these bodies. A CCD has been used in scanning mode in the University of Arizona's 'Spacewatch' program for the discovery of minor planets since as long ago as 1984 (Gehrels 1984, Gehrels et al. 1986), while a CCD in stare mode was first applied as a matter of routine to an established observing program for astrometric follow-up in 1989 - that at the Oak Ridge Observatory in Massachusetts (McCrosky 1990). After its initial 1984-1986 success, Spacewatch was modified with the help of a larger CCD and improved computer software and with the adoption of the particular mission of searching for NEOs, or minor planets (and comets) that pass close to the earth (Rabinowitz 1991, Scotti 1994). The Oak Ridge program utilizes a $1.5-\mathrm{m}$ reflector, and the first CCD observations were reduced using the Astrographic Catalogue, the mainstay of the Oak Ridge photographic program back to its inauguration in 1972, as well as of other older photographic programs in which the fields observed were significantly less than $1^{\circ}$ across. Within months, the availability, on CD-ROMs, of the STScI Guide Star Catalogue (Villard 1989) effectively consigned the venerable $A C$ to the scrap-heap, and the rapid development of ready-made and relatively inexpensive CCD systems (e.g., di Cicco 1992) has recently increased the volume of CCD astrometry considerably, allowing it to be conveniently and reliably carried out, even by amateur astronomers. At the present time, very nearly 50 percent of the as- 
trometric observations, typically 6000 , published each month in the Minor Planet Center's Minor Planet Circulars are obtained by means of a CCD.

\section{The growth of discoveries}

In an earlier review, prepared for the Asteroids II conference held early in 1988 (Bowell et al. 1989), there was some discussion of the exponentiality of the growth of discoveries of minor planets. Table I in that review showed that, except for one enormously high and four very low anomalies, the doubling time between 1854 , when there were $2^{5}$ known minor planets, and 1979 , when the number that had been given designations reached $2^{15}$, was consistently in the range 6-18 years. From recent patterns it was predicted that the number would not reach $2^{16}$ until 1993 . In fact, this number was achieved in 1991, the latest doubling interval being close to the mean of all the doubling intervals.

At present the number of designations is 93032 . This is the total number of post-1925-style MPC provisional designations, augmented by those in the Palomar-Leiden and three Palomar-Leiden Trojan surveys, as well as by the 1053 objects that had received their permanent numbers prior to $1925-$ although the number needs to be reduced by 552 for designations that have been removed either before or after publication. This suggests that $2^{\mathbf{1 7}}$ may be reached by the end of the century, but the change from photography to CCD and the possibility that some extensive 'Spaceguard' program (e.g., Morrison et al. 1992) will be initiated to search for NEOs render this very uncertain.

In any case, the count is becoming meaningless because of the new practice of generally not giving designations to objects observed on a single night. One of the proposals made in the Asteroids II paper was to downgrade the status of these 'one-night stands'. The initial plan was that they would simply be held over in anticipation that other observers might independently record the objects on two or more nights and thus justifiably claim credit for a more useful discovery. If there were no independent discoveries over four to six months, say, the one-night stands would then receive designations.

In practice, after 1988 this waiting period got longer and longer, and since mid-1992 it has become infinite. Single-night observations of Spacewatch discoveries have generally not been given designations since the start of this project, and they are currently being made at a rate in excess of 60000 per year! All the single-night data do automatically remain in the MPC files, and from time to time they resurface as isolated additional observations of objects newly identified at more than one opposition. 


\section{Numbered minor planets}

In the Asteroids II review there was the remark that "it is our premise that the aim of asteroid discovery is to augment the set of numbered asteroids, and that this set should comprise asteroids with orbits that are sufficiently well determined, not only to provide a satisfactorily detailed and reasonably unbiased delineation of the structure of the whole asteroid belt, but also to permit the ready observation of a specific member at any subsequent time." At that time there were 3774 numbered minor planets, 90 percent of which were routinely predictable to 4 arcsec or better; two of the numbered planets, (719) Albert and (878) Mildred, were lost. Now there are 6465 numbered minor planets, well over 95 percent of which are predictable to 2 arcsec or better, and (878) Mildred has been found (Williams 1991).

The mean doubling rate for the numbering of minor planets has been 15.7 years, although variations from the mean have been rather greater than for the discovery growth as a whole. The prediction that there would be $2^{12}$ numbered minor planets by 1989.8 required correction by -0.4 year, giving a doubling interval of only 11.2 years, the smallest since the 6.4 years for doubling from $2^{5}$ to $2^{6}$. Despite ever more stringent requirements for the numbering of a minor planet, it is now anticipated that the next doubling will take less than nine years, with $2^{13}$ perhaps being attained by the end of 1997. In the batch of $M P C$ s for April 1995 there were a record 77 new numberings, followed by 76 more a month later.

The lion's share of the numbered minor planets has come from the photographic search program carried out since 1963 by N.S. Chernykh and his associates with the 0.4-m astrograph at the Crimean Astrophysical Observatory. With 832 numbered objects, the CrAO program took the lead from the old Heidelberg program, with 809 numbered objects since its inception in 1891, near the end of 1994. It was suggested (Marsden 1994a) that the numbered discoveries at Palomar (which consist mainly of the Palomar-Leiden surveys of Gehrels and the van Houtens with the 1.2-m Schmidt and the NEO patrols by Helin and the Shoemakers, mainly with the 0.46-m Schmidt) would move into first place "rather soon" after CrAO had surpassed Heidelberg. This has not yet happened, the Palomar score currently being 782 , but it should be noted that, during the two years since that earlier review was prepared, Heidelberg's total has increased by 0.9 percent, CrAO's by 9.5 percent and Palomar's by as much as 34.4 percent.

After Palomar takes the lead, it is unlikely that there will be new contenders for some considerable time. Fourth-place Lowell Observatory is considerably behind the first three, and essentially all of the principal photographic patrols have now terminated or are in the process of winding down their operations. The Heidelberg and Lowell programs ended in 1959 
and 1988, respectively, and there has been little activity from the CrAO program since 1990. The various Palomar-Leiden surveys took place during 1960-1977, the Shoemaker program stopped in late 1994, and Helin is curtailing her program in favor of developing a CCD survey in Hawaii.

The leading nineteenth-century visual search program had been surpassed by the Heidelberg photographic program after the latter had been in operation for little more than a decade, and within another five years more numbered minor planets had been discovered photographically than visually. However, the influence of the CCD revolution is - at least for the present-less extreme. The small fields of the current CCD programs, even Spacewatch, mean that new numberings are going to be dominated by the backlog of identifications of older photographic discoveries for some considerable time to come. There are already 4792 multiple-opposition objects, almost all of them photographic discoveries, that can be numbered when enough additional observations have been made that their orbit solutions are considered to qualify. At present there are 33 CCD discoveries among the numbered minor planets-still a small number, but one that has shown a fourfold increase during the past two years.

\section{Qualification for numbering}

The introduction of a system of provisional designations in 1892 was intended simply to relieve the pressure caused by the rapid increase in discovery rate brought about by the introduction of photography. Almost all of the objects designated did get numbered, only a short time later, and usually even in the order the provisional designations had been applied. Occasionally the number was given before it was appreciated that the object was already known and had a number, so the new number was then simply reused. From time to time an identification with an earlier object, numbered or unnumbered, was recognized while the new object still just had a provisional designation, but in general it was felt that an object could be numbered if there were an orbit solution from observations made over a month or two. Interestingly, the orbits of the three famous objects of the 1930s that were found to cross the orbit of the earth were not judged good enough to permit numbering. Earnest attempts were made to recover numbered minor planets at their next favorable oppositions, but this became progressively more difficult, and by the time of World War II some 6 percent of the 1500 or so numbered objects had still been observed only at their discovery oppositions, often decades earlier.

On establishing the MPC in 1947 then-director Paul Herget declared a moratorium on new numberings, while efforts were directed instead to reobserving those already numbered. Numbering was resumed after the IAU 
General Assembly in 1948, when it was resolved that objects observed at a single opposition could be numbered only if they passed closer to the sun than the aphelion of Mars, but the orbit solutions for Apollo, Adonis and Hermes were still considered too weak to allow them to qualify. Actually, the concession for Mars crossers was made only three times-for (1565) Lemaitre, (1566) Icarus and (1580) Betulia-because Herget wanted to ensure that further numbered minor planets would never be lost, an aim the MPC has so far completely met. Largely unwritten further tightening of the rules later meant that two oppositions became the minimum for a Mars crosser, while for more distant objects the basic requirement was usually three good oppositions or two good and two poor oppositions.

In 1991 the MPC adopted a more quantitative guideline for deciding when a minor planet should be numbered. Among the most important components in the decision are the number $N$ of oppositions at which observations are utilized in the orbit solution and the total span of time covered by them. This time span is represented by the number $M$ of completed decades between the first and last observations, although isolated observations on a single night at the first opposition are ignored, and $M$ is restricted to a maximum of 4 . Next it is necessary to consider the distribution of the observations at each opposition. This is done by assigning, for each opposition, an integer between 0 (for single-night observations) and 8 (essentially for observations on two or more nights in each of three lunations); the sum of the scores at the individual oppositions is denoted by $L$. The distribution of observations around the orbit is then considered by looking for the longest consecutive run of calendar months in which there are no observations at any of the oppositions used in the computation of $M$; the relevant quantity $K$ ranges between 0 and 6 , the extremes being the cases when all the observations are in the same month and when there are observations in every month. The sum $J=K+L+M+N$ is then formed. Numbering is considered if all of the following are true: (a) the residuals are reasonable, the determination of $J$ of course being modified if critical observations are not good enough for inclusion in the orbit computation; (b) the latest observations in the solution are no more than two years old; (c) the contribution to $L$ at the latest opposition is 4 or more; (d) $N \geq 4$ (although $N=3$ is allowed if each contribution to $L$ is 6 or more, and $N=2$ is allowed if, in addition, the perihelion distance $<1.3 \mathrm{AU})$; and (e) $J \geq 21$, critical discussion being necessary if $J<23$.

The above criterion has the advantage that the initial selection of objects for consideration for numbering can be made automatically in the absence of orbital solutions. Muinonen and Bowell (1993) devised a more sophisticated criterion, based on Bayesian probability, which has a generally similar outcome, except for objects that pass very close to the earth, 
and they did not consider that the latest observations should have been made quite recently. They did remark that they did not think (5209) 1989 $\mathrm{CW}_{1}$ should have been numbered. This object was a Trojan that had been well observed at three oppositions and scored $J=23$. The point is that the observations therefore covered only one-sixth of an orbital revolution, and the motion can thus scarcely be considered very extrapolable. This was known to be a weakness of the MPC system, but if the Muinonen-Bowell criterion is followed, more than a century will elapse before any of the transneptunian objects found during the past few years can be numbered!

Nevertheless, the MPC has recently supplemented its criterion to address the Trojan case more satisfactorily. It has done this by equating the formal mean error in the determination of the orbital semimajor axis to the error in the position in the heliocentric orbit after one decade, this being expressed by another integer $U$ on a logarithmic scale such that an error of less than 1 arcsec gives $U=0$ and that $U=9$ means that the error approaches half a revolution (and more). Numbering is considered only if $U \leq 2$. Further, if $N<4$, numbering requires $U \leq 1$. This effectively restricts three-opposition numberings to NEOs, a very occasional one qualifying at only two oppositions.

It is useful to introduce checks from time to time to ensure standards in the set of numbered minor planets. A "critical list" is maintained of objects that have not been observed during the past ten years or at fewer than four oppositions in all. Two recent categories added in the version issued by the MPC indicate objects observed on only a single night during the past ten years and objects for which new orbit solutions show that they would fail the current stringent numbering standards.

\section{Processing the observations}

Although photography may be on the way out, some of the photographic surveys for minor planets provide excellent examples of efficient ways to discover and follow-up main-belt minor planets.

Ideally, wide-field exposures are made of an ecliptic field on two neighboring nights shortly before opposition. Images of the same minor planets are recognized on the two nights and their measurements e-mailed to the MPC utilizing a unique observer-defined designation for each object. It is not necessary for the observer actually to identify the object. Obvious identifications are automatically made at the MPC, and orbit improvements are computed as necessary. Unidentified two-night detections are given new MPC designations, and the discovery information is recorded.

Väisälä orbits are next computed and filed as convenient representations of these objects, available for use in processing the next set of observations 
to be reported. Such orbits, which involve the assumption that the objects are at perihelion (or at aphelion) can be established for a variety of presumed distances, although it is reasonable to adopt one suitably representative example for each object. The concept, devised for use with the old observing program at Turku (Väisälä 1939), is arguably the most important twentieth-century aid to the study of unperturbed orbits. The night-pairs of unidentified objects can be compared with all the existing orbits (even quite poor orbits) computed from observations at single oppositions in the past, searching for cases where a simple shift in the mean anomaly gives a close match. Attempts to verify these suggested identifications can then be made, with genuine new multiple-opposition orbits thereby emerging for probable eventual numbering.

The observer then obtains similar exposures on two nights a month later of the appropriately shifted field, makes measurements of all minor planets found and communicates them as before. Although many of the objects recorded the previous month are expected to be in the field (and at about the same brightness), the observer should again only indicate which observations belong to particular objects observed on the two nights. The MPC makes the identifications using the orbits in its files, including now the Väisälä orbits computed the previous month. Possible matches between the observations of new objects in the two months can be tested for linkage. The resulting single-opposition orbits are then tested against all the remaining night-pairs of unidentified objects at previous oppositions, again looking for matches corresponding to a simple shift in mean anomaly. Possible identifications are then examined in detail, resulting new multipleopposition orbits again going into the file. Remaining unlinked night-pairs are compared with the one-opposition orbit database as before.

The above procedure of what might be termed contingent follow-up is elegant in its efficiency and pre-planning. Of course, some potential discoveries are lost, because they happen to be outside the field, either on the confirming nights of the pairs, or from one month to the other. This is particularly true when the observations are made with small-field telescopes. For small-field work, notably that nowadays involving CCD observations, new discoveries are-in the absence of identifications found using the orbit files-specifically followed up on several nights throughout the first month and into the second. A good single-opposition orbit should then be available for possible identification with past night-pairs of observations. This targeted follow-up may be more likely to lead to success with some particular object, but it is generally less efficient, given that the aim is to produce multiple-opposition orbits for eventual numbering. Targeted follow-up is further necessary to record well past opposition the objects for which the month-arc orbits do not yield identifications. If the arc over which an object 
is observed extends over the several months to evening quadrature (bearing in mind that there will be fading during this time), there is the chance that further targeted observations can directly yield the recovery of the object at a future opposition.

\section{Near-earth objects}

Discoveries of NEOs have traditionally been subject to targeted follow-up. This is understandable, because most of these NEOs were already quite near the earth at discovery, and their apparent motions thus tended to be quite different from those of other minor planets in the field. The proposed Spaceguard survey (Morrison et al. 1992) was distinctive in that it was designed to use large-aperture, large-field telescopes to find NEOs near opposition in the outer parts of their orbits (where they spend most of their time) and to rely mainly on contingent follow-up.

Recently, the Shoemaker Committee, charged by the U.S. Congress to update the Spaceguard proposal, has pointed out that the forthcoming availability of large-format, high-quantum-efficiency, fast-readout CCDs is making it feasible to carry out the Spaceguard task more quickly by means of monthly all-sky surveys for NEOs with a relatively small telescope in each hemisphere (Shoemaker et al. 1995). Certainly, all-sky coverage will make it possible to record NEOs that have their aphelia at or only slightly beyond the earth's orbit. By concentrating on opposition searches, it seems likely that objects with aphelia at $1.2 \mathrm{AU}$ have been undersampled by some 10 percent (Marsden 1995). The disadvantage is that all the follow-up, and probably even confirmation of the initial detection, will have to be targeted. This means that there must be extensive coordination of the activities, and the total communication effort could be quite horrendous.

A useful modification of the two-night contingent confirmation and follow-up procedure discussed in the previous section is to observe long before and long after midnight on one of the nights in order to get some parallax information (Marsden 1992). To attempt parallactic observations may be fine near opposition, but in an all-sky survey much of the sky cannot be observed for very long each night. An added problem with observations far from opposition is that there are likely to be two completely different solutions to the orbit-determination problem. Although an NEO enthusiast might be tempted to select the solution that puts the object moderately far away with, say, its aphelion at or near the orbit of the earth, chances are that the correct solution is the one that makes it a perfectly ordinary minor planet in the main belt and even farther away (Marsden 1991, 1995).

Muinonen and Bowell (1992) have addressed the special problem of orbital uncertainty when one is attempting to recover an NEO observed 
at only a single opposition. As briefly noted elsewhere (Marsden 1994b), their procedure suggests that the object 1989 ML could be found in 1992 within about $1^{\circ}$ of the nominal prediction. Searches for the object proved fruitless. In fact, an apparent new discovery of an NEO, designated 1992 WA, turned out to be $1989 \mathrm{ML}$, but it was some $28^{\circ}$ from the prediction!

TABLE 1. $1989 \mathrm{ML}=1992 \mathrm{WA}$

\begin{tabular}{lrllll}
\hline \multirow{2}{*}{1989} & UT & R.A. & Decl. & R.A. & Decl. \\
\hline June & 6.39 & $0.3-$ & $0.4+$ & $0.1-$ & $0.4+$ \\
& 6.41 & $0.3+$ & $0.4-$ & 0.0 & $0.4-$ \\
& 29.38 & $1.6-$ & $0.8+$ & $0.7-$ & $1.9+$ \\
& 29.41 & $0.3+$ & $3.0-$ & $0.6+$ & $2.0-$ \\
& 30.37 & $0.1-$ & $1.3+$ & $0.6-$ & $0.2-$ \\
& 30.40 & $2.0+$ & $1.7+$ & $0.8+$ & $0.1+$ \\
July & 3.34 & $1.3-$ & $0.6+$ & $(7.1-$ & $9.0-)$ \\
& 3.37 & $0.6+$ & $1.3-$ & $(5.9-$ & $11.0-)$ \\
\hline
\end{tabular}

Table 1 shows the $(\mathrm{O}-\mathrm{C})$ residuals of the 1989 observations, which were pairs made on four nights. The first set of residuals is that of a "standard" fit, treating each observation of equal weight. The $3^{\prime \prime}$ declination residual on June 29.41 is not unusual for photographic measures of trailed images of an NEO on the night of its discovery. Straight application of the MuinonenBowell theory would be naive, however, for while it may seem fortunate to have the June 6 prediscovery observations, they are quite isolated in time from the others. From the point of view of error assessment, it would therefore be quite appropriate completely to discount them. In that case there would be only a four-night arc of observations, and it would be inappropriate to expect the object to be found in 1992.

The second set of residuals in Table 1 is from a fit to the 1989 and 1992 observations combined. This fit shows that the problem was not in fact with the June 6 observations, but with those on July 3 , at the end of the four-day post-discovery arc. This outcome may seem a little unexpected, for these observations were not really isolated in time. But this is again a symptom of the isolation of the June 6 measures, even though those measures turned out to be very good. Independent observations on a second night in early June would immediately have shown something to be wrong, and with only a little experimentation, attempts to fit those early-June data with the observations on two of the nights during June 29-July 3 would have shown, not only that the problem was on July 3 , but also have provided confidence that the solution from the other night-pairs was dependable. 


\section{Transneptunian objects}

The transneptunian objects (TNOs) discovered since 1992 have been no brighter than 22nd magnitude, and the difficulty of obtaining the necessary follow-up in order to determine definite orbital solutions is extreme. The first two years of this effort, involving 13 objects, has been reviewed elsewhere (Marsden 1994c). While bearing in mind that there is always the possibility that some of the transneptunians are objects in unstable planet-crossing orbits detected near aphelion, it seemed that the discoveries were almost equally divided into two groups. One group consisted of loweccentricity, low-inclination objects with semimajor axes $a=42-46 \mathrm{AU}$, their essential orbital stability ensured by the impossibility of approaches within $10 \mathrm{AU}$ of Neptune. The second group seemed to contain objects discovered much closer to Neptune's orbit but prevented from encountering Neptune, again generally within $10 \mathrm{AU}$, because their orbits are librating about the 2:3 resonance with Neptune-i.e., they are like Pluto and have $a=39-40 \mathrm{AU}$; some of these orbits have rather moderate eccentricities and inclinations, up to 0.32 (for $1993 \mathrm{SB}$ ) and $17^{\circ}$ (for Pluto), and approaches within only some $8 \mathrm{AU}$ of Uranus are possible.

Table 2 lists the 28 TNOs discovered between mid-1992 and mid-1995. $H_{\mathrm{R}}$ is the absolute red magnitude, and the next six columns give the usual orbital elements (in standard notation) for the common epoch 1995 Mar. 24. The column labeled 'Arc' shows the span in days between the first and last observations, an asterisk indicating that there are observations at additional oppositions. The general pattern noted previously still holds, although the fraction suspected as being 2:3 Neptune librators is now somewhat less than half; only one more multiple-opposition object, $1994 \mathrm{JR}_{1}$, has been added to the three previously established (more or less) as being in the resonance.

A few more features have developed in the distribution. One is represented by $1995 \mathrm{DA}_{2}$, an object found opposite Neptune but only just beyond its orbit. It was immediately recognized that at this could not be a stable 2:3 librator, so the possibility of 1:2, 3:4 and even 3:5 librations was considered instead. Although there can be no proof from an orbit with an orbital arc of only 37 days, a 3:4 solution was adopted that seems to keep the object more than $10 \mathrm{AU}$ from Neptune. Rather more conclusive is the case of $1994 \mathrm{JS}$, initially suspected as being a 2:3 librator, a possibility that has clearly been disproven by the observations in 1995. It seems very probable that $1994 \mathrm{JS}$ is instead a 3:5 librator, its minimum distance from Neptune being $10 \mathrm{AU}$. An attempt at forcing $1995 \mathrm{DA}_{2}$ to be a 3:5 librator gave a similar minimum distance.

Of the 13 TNOs discovered by mid-1994 two, 1993 RP and $1994 \mathrm{JV}$, failed to be observed at their next opposition. Both have been considered 
TABLE 2. Transneptunian objects

\begin{tabular}{|c|c|c|c|c|c|c|c|c|c|}
\hline Object & $H_{\mathrm{R}}$ & $M$ & $\omega$ & $\Omega$ & $i$ & $e$ & $a$ & Arc & $M P C$ \\
\hline $1995 \mathrm{DA}_{2}$ & 7.6 & 309.38 & 62.35 & 127.49 & 6.58 & 0.1156 & 36.3447 & 37 & 25184 \\
\hline $1993 \mathrm{RO}$ & 8.0 & 357.03 & 187.46 & 170.30 & 3.72 & 0.1982 & 39.2782 & $*$ & 24241 \\
\hline $1993 \mathrm{RP}$ & 9.0 & 2.22 & 180.64 & 192.09 & 2.57 & 0.1135 & 39.3289 & 2 & 23493 \\
\hline $1993 \mathrm{SB}$ & 7.5 & 319.73 & 79.12 & 354.81 & 1.93 & 0.3216 & 39.3897 & $*$ & 24408 \\
\hline 1994 JV & 7.0 & 1.25 & 179.99 & 28.15 & 16.48 & 0.1247 & 39.4570 & 25 & unpub. \\
\hline $1995 \mathrm{GA}_{7}$ & 7.2 & 63.86 & 100.20 & 20.97 & 3.54 & 0.1192 & 39.4551 & 2 & 25186 \\
\hline $1993 \mathrm{SC}$ & 6.3 & 33.90 & 319.22 & 354.64 & 5.16 & 0.1795 & 39.4708 & $*$ & 24763 \\
\hline $1995 \mathrm{KK}_{1}$ & 7.8 & 21.54 & 328.35 & 228.09 & 9.25 & 0.1898 & 39.4748 & 1 & 25315 \\
\hline $1995 \mathrm{HM}_{5}$ & 7.9 & 3.26 & 354.78 & 186.70 & 4.60 & 0.1775 & 39.5337 & 33 & 25315 \\
\hline 1994 ТВ & 6.5 & 31.41 & 340.17 & 316.89 & 11.98 & 0.2917 & 39.5656 & 37 & 25184 \\
\hline $1994 \mathrm{JR}_{1}$ & 7.1 & 5.73 & 91.98 & 144.72 & 3.80 & 0.1277 & 39.8261 & $*$ & 25341 \\
\hline $1994 \mathrm{TH}$ & 6.9 & 0.7 & 356.62 & 12.12 & 16.07 & 0.0 & 40.9404 & 3 & 24084 \\
\hline $1995 \mathrm{GY}_{7}$ & 7.5 & 0.0 & 203.47 & 34.50 & 0.94 & 0.0 & 41.3469 & 1 & 25408 \\
\hline $1994 \mathrm{TG}$ & 6.7 & 0.6 & 353.02 & 15.51 & 6.76 & 0.0 & 42.2544 & 3 & 24084 \\
\hline $1995 \mathrm{FB}_{21}$ & 7.5 & 0.0 & 209.63 & 28.36 & 0.68 & 0.0 & 42.4259 & 9 & 25408 \\
\hline $1994 \mathrm{TG}_{2}$ & 7.7 & 0.4 & 358.86 & 353.28 & 2.25 & 0.0 & 42.4479 & 32 & 24884 \\
\hline $1994 \mathrm{JS}$ & 6.8 & 323.68 & 238.18 & 56.33 & 14.03 & 0.2343 & 42.8408 & $*$ & 25341 \\
\hline $1995 \mathrm{GJ}$ & 6.5 & 359.96 & 180.29 & 338.93 & 22.93 & 0.0909 & 42.9072 & 1 & 25185 \\
\hline $1994 \mathrm{EV}_{3}$ & 6.8 & 165.26 & 13.81 & 19.51 & 1.68 & 0.0381 & 43.0703 & $*$ & 25228 \\
\hline $1995 \mathrm{KJ}_{1}$ & 6.1 & 359.8 & 180.40 & 47.96 & 3.80 & 0.0 & 43.2345 & 1 & 25315 \\
\hline $1994 \mathrm{VK}_{8}$ & 5.9 & 0.3 & 349.03 & 72.83 & 1.43 & 0.0 & 43.4504 & 86 & 24715 \\
\hline $1995 \mathrm{DB}_{2}$ & 6.4 & 0.10 & 0.16 & 128.64 & 4.27 & 0.0671 & 43.4935 & 34 & 25031 \\
\hline $1994 \mathrm{GV}_{9}$ & 6.8 & 20.84 & 335.24 & 176.85 & 0.55 & 0.0420 & 43.7308 & $*$ & 25228 \\
\hline $1993 \mathrm{FW}$ & 6.6 & 322.72 & 43.26 & 187.92 & 7.74 & 0.0521 & 43.8644 & $*$ & 25227 \\
\hline $1992 \mathrm{QB}_{1}$ & 6.7 & 5.80 & 357.35 & 359.40 & 2.19 & 0.0698 & 43.9389 & $*$ & 24408 \\
\hline $1994 \mathrm{JQ}_{1}$ & 6.5 & 334.80 & 211.40 & 25.65 & 3.74 & 0.0267 & 44.1922 & $*$ & 25228 \\
\hline $1995 \mathrm{DC}_{2}$ & 6.0 & 0.1 & 358.78 & 154.29 & 2.11 & 0.0 & 45.2076 & 42 & 25184 \\
\hline $1994 \mathrm{ES}_{2}$ & 7.6 & 279.84 & 99.95 & 154.73 & 1.05 & 0.1333 & 45.9596 & $*$ & 25341 \\
\hline
\end{tabular}

as 2:3 librators, although, following recognition of the altered status of 1994 JS, searches-the outcome of which is still not entirely conclusivefor $1994 \mathrm{JV}$ were also made in 1995 on the assumption that its orbit is in $3: 5$ resonance. A recovery of the 14 th TNO, $1994 \mathrm{~TB}$, has recently been made (Gladman 1995), but further observations will be needed before one can state whether this object is a 2:3 librator, a 3:4 librator or has an orbit that is unstable.

Table 2 shows two weak circular orbits around $a=41$ AU. It is likely that these objects, $1994 \mathrm{TH}$ and $1995 \mathrm{GY}_{7}$, are "outer group" objects near perihelion. Duncan et al. (1995) have expressed surprise that there seem 
not to be objects in non-resonant, low-eccentricity orbits with $a=37-38$ $\mathrm{AU}$ that could be stable. Certainly, no objects have yet been established as in such orbits, although it is not impossible that $1995 \mathrm{GA}_{7}$ is a candidate.

\section{References}

Bowell, E., Chernykh, N.S. and Marsden, B.G., 1989. Discovery and follow-up of asteroids. In Asteroids II, eds. R.P. Binzel, T. Gehrels and M.S. Matthews, University of Arizona Press, Tucson, pp. 21-38.

di Cicco, D., 1992. The ST-6 Imaging Camera, Sky Tel., 84, 395-401.

Duncan, M.J., Levison, H.F. and Budd, S.M., 1995. The dynamical structure of the Kuiper Belt, in press.

Gehrels, T., 1984. Observations made with the Spacewatch Camera 0.91-m telescope at Kitt Peak, Minor Planet Circ., No. 9198.

Gehrels, T., Marsden, B.G., McMillan, R.S. and Scotti, J.V., 1986. Astrometry with a scanning CCD, Astron. J., 91, 1242-1243.

Gladman, B., 1995. 1994 TB. Minor Planet Electronic Circ., 1995-M07.

Marsden, B.G., 1991. The computation of orbits in indeterminate and uncertain cases, Astron. J., 102, 1539-1552.

Marsden, B.G., 1992. Comments on search programs for near-earth objects. In Observations and physical properties of small solar system bodies (30th Liège Internat. Astrophys. Colloq.), eds., A. Brahic, J.-C. Gérard and J. Surdej, Université de Liège, pp. 251-252.

Marsden, B.G., 1994a. Asteroid and comet surveys. In Astronomy from Wide-Field Imaging (IAU Symposium No. 161), eds. H.T. MacGillivray, E.B. Thomson, B.M. Lasker, I.N. Reid. D.F. Malin, R.M. West and H. Lorenz, Kluwer Academic Publishers, Dordrecht, pp. 385-399.

Marsden, B.G., 1994b. Astrometric observations of minor planets and comets: present and future needs. In Galactic and Solar System Optical Astrometry, eds. L.V. Morrison and G.F. Gilmore, Cambridge University Press, pp. 263-275.

Marsden, B.G., 1994c. Searches for planets and comets. In Inventory of the solar system (Astron. Soc. Pacific Conference Series), in press.

Marsden, B.G., 1995. Overview of orbits. In International conference on Near-Earth Objects, ed. J. Remo, N.Y. Academy of Sciences, New York, in press.

McCrosky, R.E., 1990. 801 Oak Ridge, Minor Planet Circ., No. 15630.

Morrison, D. et al., 1992. The Spaceguard Survey, NASA, Washington.

Muinonen, K. and Bowell, E., 1992. Orbital uncertainties for earth-crossing asteroids, Bull. Am. Astron. Soc., 24, 942.

Muinonen, K. and Bowell, E., 1993. Asteroid orbit determination using Bayesian probabilities, Icarus, 104, 255-279.

Rabinowitz, D.L., 1991. Detection of earth-approaching asteroids in near real time, Astron. J., 101, 1518-1529.

Scotti, J.V., 1994. Computer aided near earth object detection. In Asteroids, Comets, Meteors 1993 (IAU Symposium No. 160), eds. A. Milani, M. di Martino and A. Cellino, Kluwer Academic Publishers, Dordrecht, pp. 17-30.

Shoemaker, E.M. et al., 1995. Report of the near-earth object survey working group, NASA, Washington.

Väisälä, Y., 1939. Eine einfache Methode der Bahnbestimmung, Mitt. Sternw. Univ. Turku No. 1.

Villard, R., 1989. The world's biggest star catalogue, Sky Tel., 78, 583-589.

Williams, G.V., 1991. (878) Mildred, IAU Circ., No. 5275. 